\title{
Health Telemonitoring
}

National Cancer Institute

\section{Source}

National Cancer Institute. Health Telemonitoring. NCI Thesaurus. Code C118567.

The use of video, audio, and other methods of telecommunicating to monitor a patient's health status from a site distant to the patient. 